\title{
Le Xeroderma pigmentosum : caractéristiques cliniques, génétiques et cellulaires Développement d'un test anténatal
}

Le Xeroderma pigmentosum est une maladie autosomique récessive caractérisée cliniquement par l'hypersensibilité de la peau au soleil et par l'apparition précoce de multiples cancers cutanés et oculaires. Le défaut biochimique est une anomalie de la réparation de l'ADN lésé par les rayonnements ultraviolets, le plus souvent en rapport avec une absence d'incision de l'ADN de la lésion. Il existe au moins dix types de modifications génétiques (définis par des groupes de complémentation différents) à l'origine de la maladie. La mise en évidence, sur des cellules en culture, de l'anomalie de réparation de l'ADN permet de faire, dans les cas typiques, le diagnostic prénatal de l'affection.

\section{Alain Sarasin Georges Renault Claudine Blanchet-Bardon Joëlle Boue Yves Dumez}

\section{ADRESSES}

A. Sarasin : directeur de recherche au Cnrs. G. Renault: ingénieur d'études au Cnrs. Laboratoire de génétique moléculaire, Institut de recherches scientifiques sur le cancer, $\mathrm{BP} \mathrm{n}^{\circ} 8,94802$ Villejuif Cedex, Françe. C. Blanchet-Bardon: directeur de recherche au Cnrs. Clinique des maladies cutanées, hôpital Saint-Louis, 75010 Paris, France. J. Boue: directeur de recherche au Cnrs. Inserm U. 73, château de Longchamp, 75016 Paris, France.

Y. Dumez: directeur de recherche au Cnrs. Maternité Port-Royal, hôpital de Port-Royal,

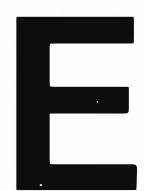

n 1870, M. Kaposi donna le nom de Xeroderma pigmentosum (XP) au syndrome présenté par une série de malades ayant en commun une peau en parchemin associée à une pigmentation hétérogène de la peau exposée au soleil. Des désordres neurologiques sont souvent associés au XP dont la forme la plus extrême, le syndrome de De Sanctis-Cacchione, est liée à une détérioration mentale progressive, à un nanisme et à un développement sexuel limité. La photophobie et les lésions oculaires représentent aussi une constante de cette maladie. Sa transmission héréditaire mise à part, la caractéristique la plus évidente du XP est le taux élevé d'épithéliomas cutanés observés dans les zones exposées au soleil. Celle-ci est à relier à la découverte de J. Cleaver [1] qui démontra que les cellules isolées des malades XP étaient particulièrement sensibles aux rayonnements ultraviolets (UV) du fait d'une absence de réparation des lésions induites sur l'ADN cellulaire. Le XP représente l'exemple le plus probant chez l'homme de l'initiation de cancers, surtout cutanés, dus à la présence de lésions non réparées sur le patrimoine génétique. Ainsi, dans la majorité des cas, la mort des malades est due aux cancers et elle survient en moyenne 30 ans plus tôt que dans la population générale.

\section{Caractéristiques cliniques}

Le XP a été décrit dans toutes les races et est transmis de façon autosomique et récessive. Sa fréquence varie de 1/1 000000 en Europe et aux États-Unis à $1 / 100000$ au Japon, en Afrique du Nord et en Égypte, où elle est liée à une consanguinité des parents : $30 \%$ des parents de XP sont consanguins. Ceux-ci, hétérozygotes pour la maladie, sont cliniquement dénués de symptômes.

Plusieurs formes de XP ont été défi- 
nies sur des considérations cliniques, puis sur des critères biochimiques et génétiques.

- XP classique. Dans cette forme, la plus commune parmi les XP, $75 \%$ des malades voient leurs symptômes apparaître entre deux et quatre ans. Les premiers correspondent à une hypersensibilité au soleil, caractérisée par un érythème sévère suivant une exposition courte et une photophobie détectable dès la naissance. La pigmentation hétérogène de la peau exposée apparaît vers l'âge de deux ans et peut être associée à une atrophie cutanée et à des télangiectasies des zones exposées incluant le visage, les lèvres et les paupières. L'association de ces troubles est appelée poïkilodermie et est similaire à celle retrouvée chez des adultes ayant subi une irradiation gamma générale. Des kératoses actiniques, des kérato-acanthomes, des ulcérations qui doivent faire craindre un cancer cutané apparaissent sur la peau exposée, y compris les paupières et la conjonctive. Les anomalies oculaires (photophobie, lésions de la conjonctive, de la cornée et des paupières) sont particulièrement nombreuses chez les malades atteints

\begin{tabular}{|l|l|l|}
\hline \multicolumn{4}{|c|}{ Tableau I } \\
\multicolumn{4}{|c|}{ CARACTÉRISTIQUES CLINIQUES DES MALADES ATTEINTS } \\
DE XERODERMA PIGMENTOSUM(1)
\end{tabular}

(1) Adapté d'après [2]. (2) Étude effectuée sur 830 patients atteint de XP. Les chiffres cités ne tiennent pas compte des malades pour lesquels l'existence de tumeurs cutanées ou celle d'anomalies oculaires ne sont pas précisées dans les réponses aux enquêtes médicales. (3) Parmi 330 malades présentant au moins une tumeur cutanée.

de XP (Tableau I).

La caractéristique de la forme XP classique est l'apparition de tumeurs malignes cutanées ou oculaires, dès l'âge de huit ans en moyenne, c'est- à-dire 50 ans plus tôt que dans la population générale (figure 1). Ainsi, la fréquence des épithéliomas basoet spinocellulaires est, à l'âge de 20 ans, 4800 fois plus forte dans le XP

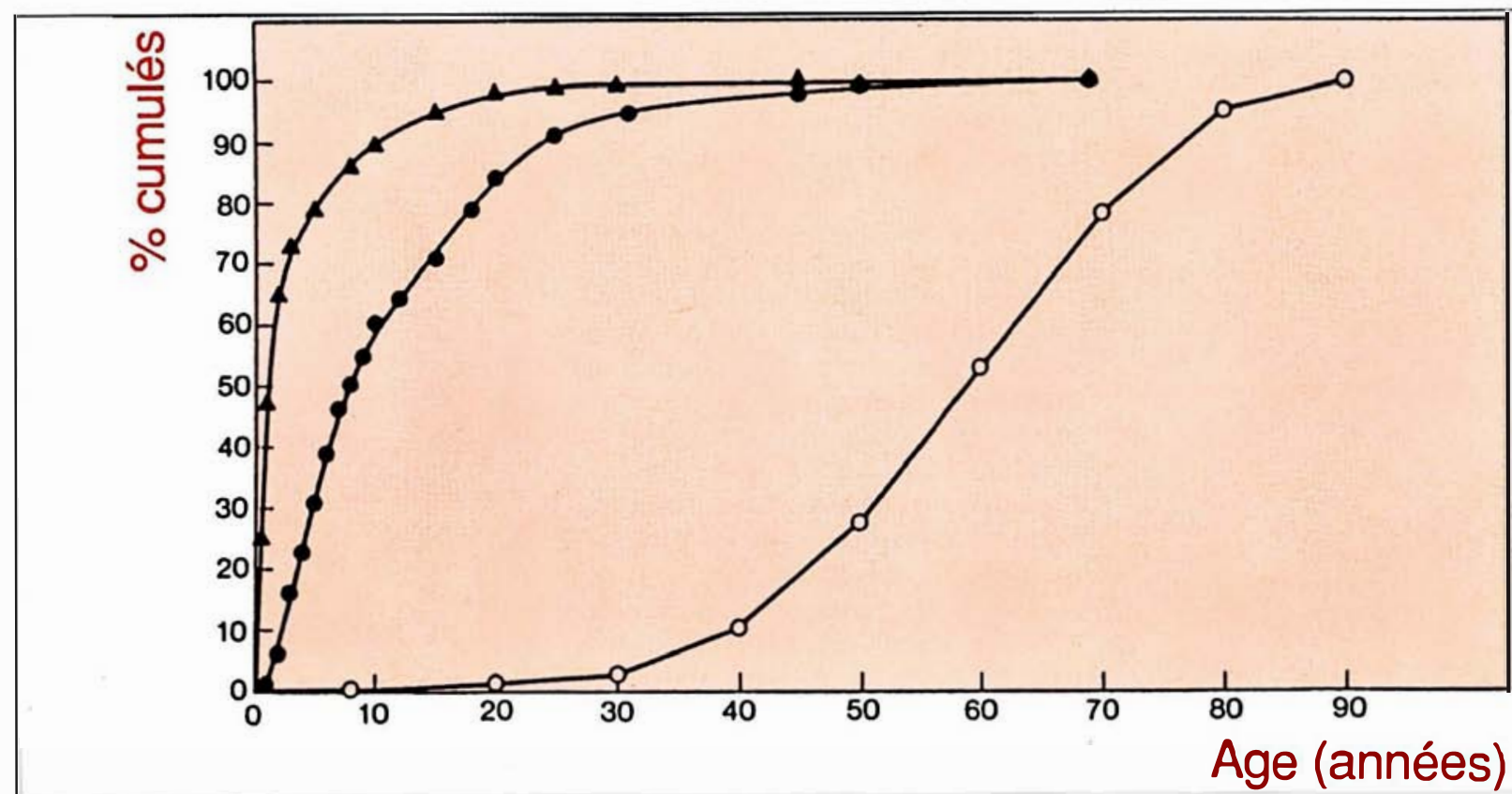

Figure 1. Age d'apparition des symptômes cutanés (hypersensibilité au soleil, hyperpigmentation) chez les patients XP ( $\triangle$ ). Age d'apparition de la première tumeur cutanée (baso- ou spinocellulaire) chez les malades XP (•) et dans la population générale américaine (0). (Reproduite d'après [2] avec permission.) 


\section{RÉFÉRENCES}

1. Cleaver JE. Defective repair replication of DNA in Xeroderma pigmentosum. Nature $1968 ; 218: 652-6$

2. Kraemer KH, Lee MM, Scotto J. Xeroderma pigmentosum. Arch Dermatol 1987; $123: 241-50$.

3. Lafforet D, Dupuy JM. Photosensibilité et réparation de l'ADN. Possibilité d'une parenté nosologique entre Xeroderma pigmentosum et syndrome de Cockayne. Arch Fr Pediatr 1978 ; 35 : 65-74.

4. Hanawalt PC, Sarasin A. Cancer prone hereditary diseases with DNA processing abnormalities. Trends Genet 1986 ; 2 : 124-9.

5. Sarasin AR, Smith CA, Hanawalt PC Repair of DNA in human cells after treatment with activated aflatoxin B1. Cancer Res 1977 37 : 1786-93.

6. Kraemer KH, Slor H. Xeroderma pigmen tosum. Clin Dermatol 1984 ; 2 : 33-69.

7. Bourre F, Renault G, Sarasin A. Sequence effect on alkali-sensitive sites in UV-irradiated SV40 DNA. Nucleic Acids Res 1987; 15 8861-75.

8. Friedberg EC. DNA repair. New York Freeman and Co, 1985 .

9. DeWeerd-Kastelein EA, Keijzer W Bootsma D. Genetic heterogeneity of Xero derma pigmentosum demonstrated by somatic cell hybridization. Nature 1972; $238: 80$ 3.

10. Wood RD, Robins P, Lindahl T. Complementation of the Xeroderma pigmentosum DNA repair defect in cell-free extracts. Cell $1988 ; 53: 97-106$.

11. Mortelmans K, Friedberg EC, Slor $\mathrm{H}$, et al. Evidence for a defect in thymine dimer excision in extracts of XP cells. Proc Natl Acad Sci USA 1976; 73 : 2757-62.

12. Bohr VA, Phillips DH, Hanawalt PC Heterogeneous DNA damage and repair in the mammalian genome. Cancer Res 1987 .

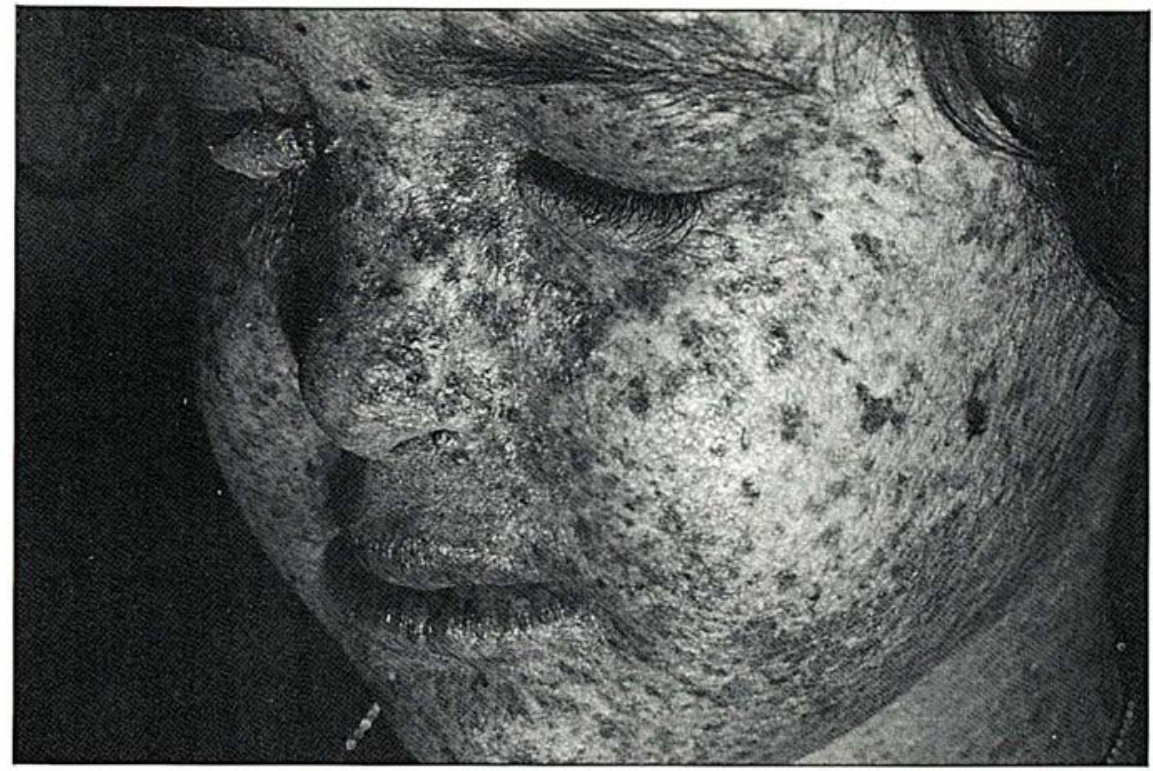

Figure 2. Xeroderma pigmentosum. Début de la maladie chez un enfant de sept ans. On peut observer, sur le visage, I'hyperpigmentation classique des XP et des débuts de formation tumorale sur le nez.

que dans un groupe témoin de la population américaine [2]. Ces tumeurs apparaissent dans $97 \%$ des cas dans les zones exposées au soleil : visage, paupières, nez, lèvres, cornée, extrémités des membres supérieurs, langue, cou et poitrine (figures 2, 3 et figure 4, p. 611). Ces tumeurs métastasent peu (14\%), mais leur multiplicité n'en est pas moins dramatique. Cinq pour cent de la population totale des malades XP développent des mélanomes malins, ce qui correspond aussi à une augmentation des risques d'environ 2000 fois par rapport à la population générale. L'âge moyen d'apparition des mélanomes (19 ans) est de dix années plus tardif que l'âge moyen d'apparition des épithéliomas. Peu de tumeurs internes ont été décrites chez le XP, toutefois les tumeurs du cerveau (médulloblastome, astrocytome) sont les plus fréquentes [2] après les tumeurs des zones exposées au soleil. Les malades avec une forme classique XP ont en général un développement statural et sexuel à peu près normal.

Dans un faible nombre de cas, la forme XP classique est associée à un retard mental progressif visible dès l'âge de 10-15 ans. La forme la plus extrême, ou syndrome de De Sanctis-

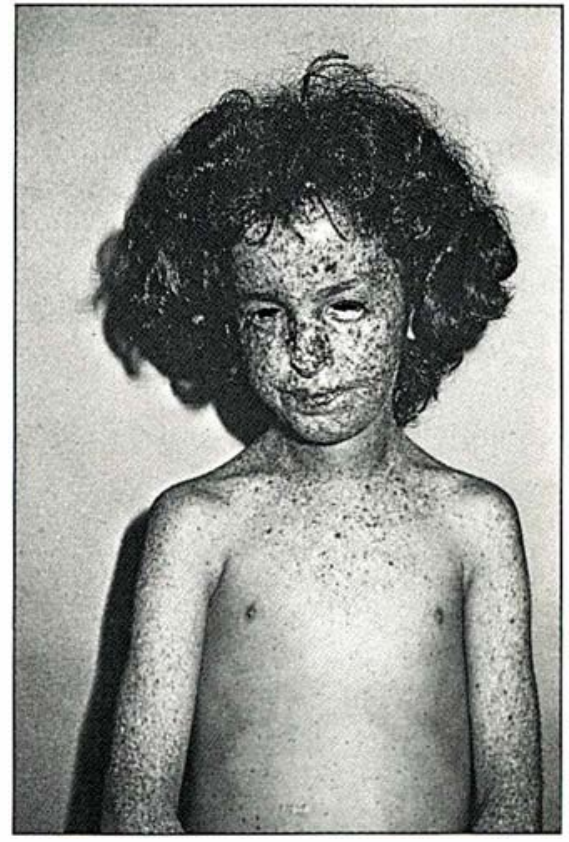

Figure 3. Xeroderma pigmentosum. Configuration typique du XP chez le scur jumelle de l'enfant de la figure 2. La peau non exposée au soleil (thorax et ventre) est pratiquement normale, tandis que la peau exposée au soleil (visage, cou et avant-bras) présente l'hyperpigmentation et de nombreuses tumeurs, en particulier sur le visage. 


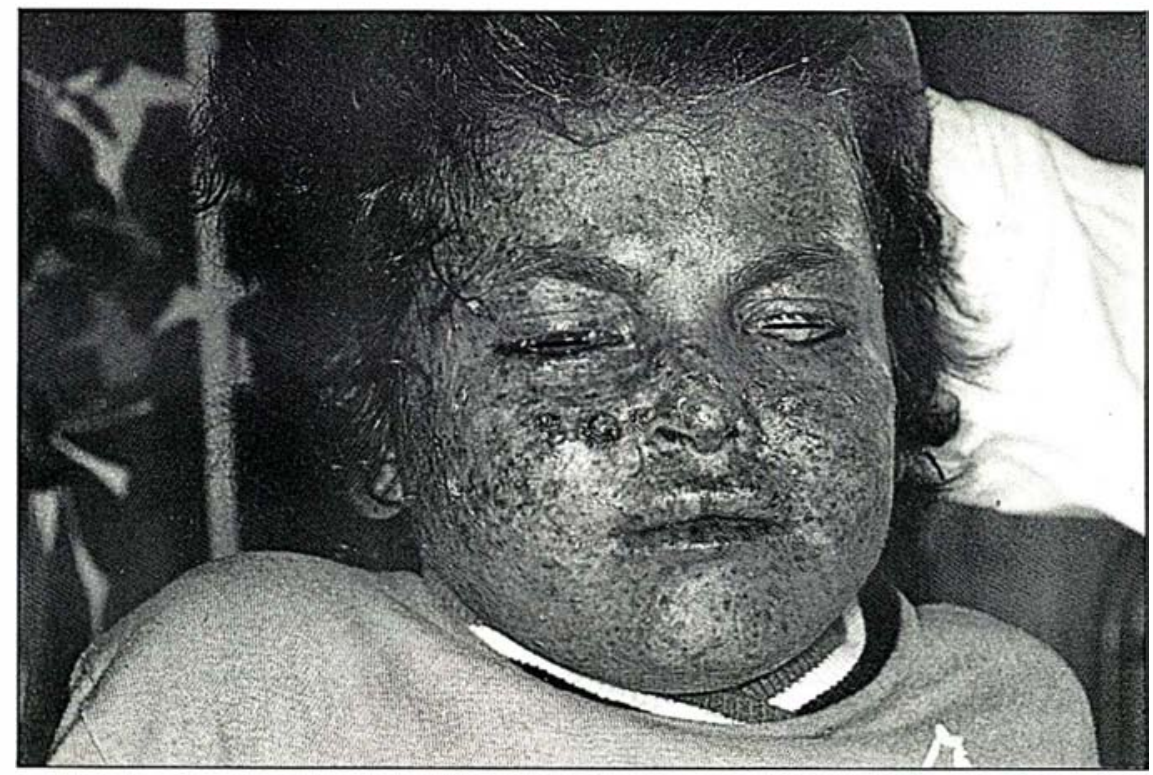

Figure 4. Xeroderma pigmentosum. Exemples de tumeurs cutanées dans les zones exposées au soleil: visage, conjonctive, cornée, paupières.
Cacchione décrite en 1932, s'accompagne de microcéphalie, d'anomalies neurologiques progressives, d'un faible quotient intellectuel, d'une hypo- ou aréflexie et d'une immaturité sexuelle.

- XP variant. Environ $20 \%$ des patients montrent plus tardivement, entre 15 et 40 ans, les signes classiques du XP. Chez ces sujets, la progression de la maladie est plus lente et l'espérance de vie est considérablement plus grande. La photophobie est souvent faible voire absente.

Le déficit biochimique et génétique des XP variants est complètement différent de celui des XP classiques (voir caractéristiques biochimiques).

- Association entre le XP et le syndrome de Cockayne. Deux patients ont été décrits possédant à la fois la maladie XP et le syndrome de Cockayne [3]. Celui-ci est une maladie héréditaire très rare (encore plus rare que le XP) transmise de façon autosomique récessive et caractérisée par un retard staturo-pondéral, une immaturité sexuelle, un retard mental, une dégénérescence pigmentaire de la rétine, un visage caractéristique en bec d'oiseau et une hypersensibi$\mathrm{m} / \mathrm{s} n^{\circ} 10$ vol. 4, décembre 88 survivre au traitement sans être mutées. Les cellules de XP sont particulièrement sensibles in vitro aux rayons UV et aux agents mimant l'effet de ce rayonnement, du fait du mauvais fonctionnement des étapes cellulaires permettant de tolérer ou d'éliminer les lésions produites $[4,5]$.

- Hypersensibilité cellulaire aux UV. Toutes les cellules de malade XP (habituellement cultures in vitro de fibroblastes de peau ou de lignées lymphoblastoïdes) ont une survie plus réduite que les cellules d'individus sains après irradiation UV. Une différence de sensibilité considérable est observée d'un malade XP à un autre, la forme XP classique étant beaucoup plus sensible que la forme variante (figure 5, p. 612). En particulier, plus les fibroblastes cultivés in vitro sont sensibles aux UV, plus la photophobie, l'apparition de tumeurs, voire les désordres neurologiques seront importants et précoces [6].

L'irradiation UV induit un nombre beaucoup plus important d'anomalies chromosomiques et d'échanges entre chromatides sœurs dans les cellules XP que dans les cellules normales. biochimiques et génétiques différentes de l'un à l'autre et des autres malades XP.

La probabilité que deux maladies héréditaires, dont les fréquences d'apparition sont si faibles, soient trouvées chez le même malade est quasi nulle. On peut donc imaginer que cette association n'est pas due au hasard, mais plutôt qu'elle est liée à une étape commune au niveau biochimique ou à une structure voisine au niveau génétique.

\section{Caractéristiques \\ biochimiques et génétiques}

Les cellules d'individus sains sont capables de tolérer un certain nombre de lésions produites sur leur molécule d'ADN par des agents chimiques (hydrocarbures polycycliques, mycotoxines, aminofluorènes, psoralènes, etc.) ou des agents physiques (rayons UV ou rayonnements ionisants). Cette tolérance dépend des systèmes de réparation spécifiques et efficaces qui permettent aux cellules endommagées de pouvoir
- Mécanismes de réparation de l'ADN. L'irradiation UV-C (254 nm de longueur d'onde) produit des lésions sur l'ADN localisées essentiellement entre deux pyrimidines adjacentes sur le même brin : les dimères de pyrimidines (Py-Py) et les pyrimidines(6-4)pyrimidones ( $\mathrm{Py}(6-4) \mathrm{Py})$ (figure 6, p. 613). La fréquence de formation de ces lésions dépend non seulement de la dose d'UV utilisée, mais aussi de la nature des deux pyrimidines impliquées et de la séquence nucléotidique adjacente [7] La quantité de Py-Py est de cinq à dix fois plus forte que celle des $\mathrm{Py}(6$ 4)Py. Ces deux lésions représentent 80 à $90 \%$ des lésions identifiées après irradiation UV. D'autres lésions, minoritaires, sont formées telles que les sites abasiques (élimination d'une des bases constituant l'ADN qui conduit à la perte locale de l'information génétique), les modifications chimiques de bases (les glycols de thymine), les pontages interbrins ou entre ADN et protéines, etc. 


\section{RÉFÉRENCES}

13. Lehmann A, Kirk-Bell S, Arlett C, et al. Xeroderma pigmentosum cells with normal levels of excision repair have a defect in DNA synthesis after UV-irradiation. Proc Natl Acad Sci USA 1975 ; 72 : 219-23.

14. Suarez HG, Nardeux P, Andeol Y, Sarasin A. Multiple activated oncogenes in human tumors. Oncogene Res 1987; 1 : 201 . 7:

15. Sarasin A, Daya-Grosjean L, Suarez HG, Chrétien B, Avril MF. Activation des oncogènes dans les tumeurs épithéliales isolées de malades atteints du Xeroderma pigmentosum. médecine/sciences $1988 ; 4: 643-7$.

16. Vuillaume M, Calvayrac R, Best-Belpomme $\mathbf{M}$, et al. Deficiency in the catalase activity of Xeroderma pigmentosum cell and simian virus 40-transformed human cell extracts. Cancer Res 1986; 46 : 538-44.

17. Halley DJJ, Keijzer W, Jaspers NGJ, et al. Prenatal diagnosis of Xeroderma pigmentosum (group C) using assays of unscheduled DNA synthesis and post replication repair. Clin Genet $1979 ; 16: 137-46$.

18. Van Duin M, DeWit J, Odijk H, et al. Molecular characterization of the human excision repair gene ERCC-1:cDNA cloning and amino acid homology with the yeast DNA repair gene RADl0. Cell $1986 ; 44: 913$ 23.

19. Sancar A, RuppWD. A novel repair enzyme: UVRABC excision nuclease of $E$. coli cuts a DNA strand on both sides of the

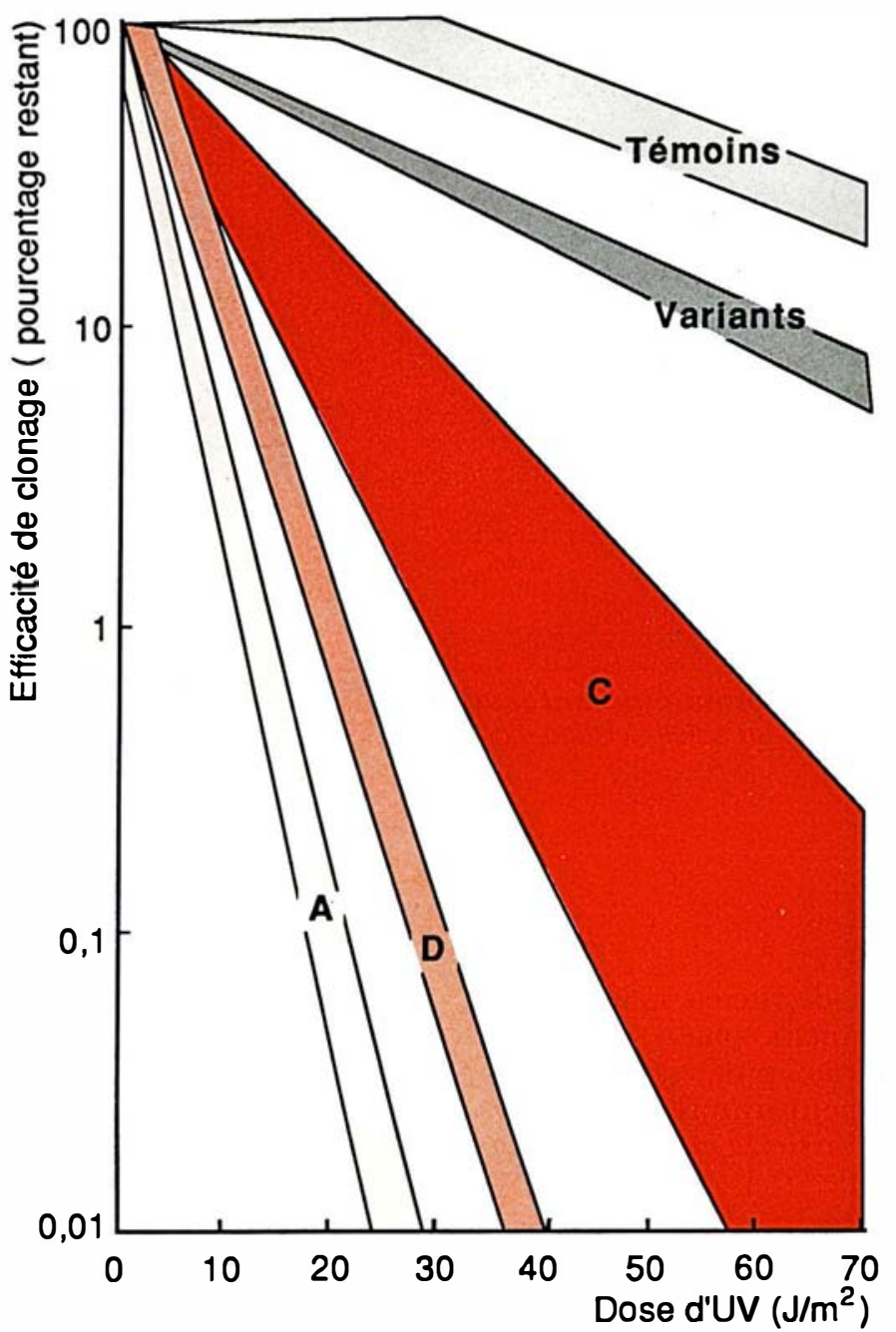

Figure 5. Survie cellulaire in vitro de fibroblastes isolés de la peau d'individus sains ou atteints de XP et irradiés avec des doses croissantes d'UV de $254 \mathrm{~nm}$. Chaque groupe de complémentation indiqué dans la figure par la lettre correspondante $(A, D, C)$ montre une sensibilité caractéristique aux UV. (Modifiée d'après [6] avec permission.)

Les lésions induites par les UV sont principalement détectées et éliminées par le système de réparation par excision-resynthèse (figure 6, p. 613). Ce processus qui fonctionne dans toute cellule vivante se compose d'une étape de reconnaissance de la lésion, probablement du fait de la déformation locale de la structure de l'ADN, suivie d'une étape d'incision du brin endommagé, puis d'excision d'un petit fragment d'ADN (entre 10 et 50 nucléotides) contenant la lésion. L'étape d'incision du brin d'ADN près de la lésion est particulièrement complexe puisqu'elle nécessite trois 


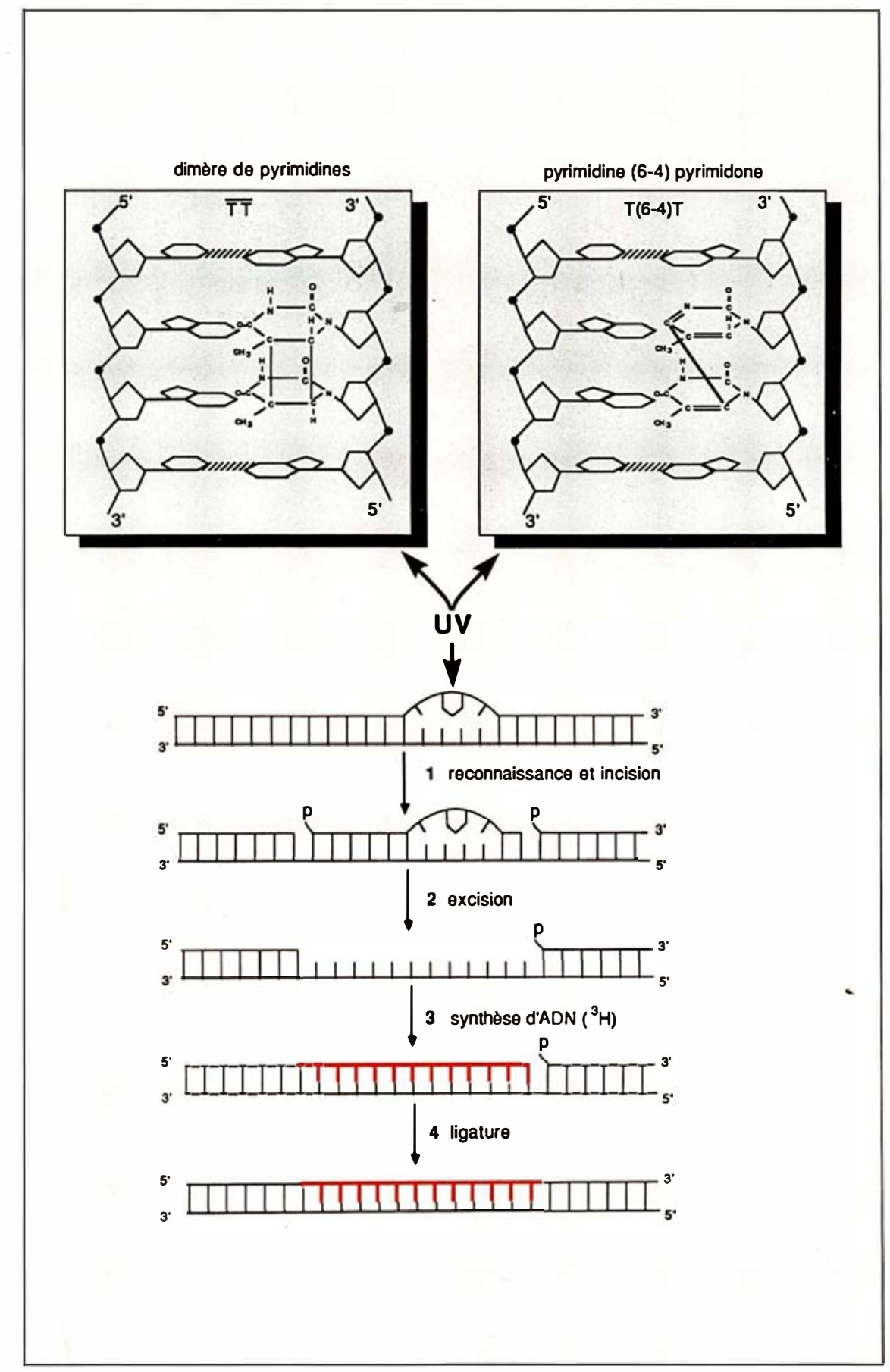

Figure 6. Structure chimique des lésions majeures induites par les UV-C $(254 \mathrm{~nm})$ sur deux pyrimidines adjacentes et représentation schématique de la voie de réparation par excision-resynthèse. (1) Étape de reconnaissance de la lésion et d'incision du brin endommagé à proximité de la lésion. (2) Excision et élimination du fragment portant la lésion. (3) Étape de polymérisation d'ADN en utilisant le brin opposé comme matrice. Le précurseur radioactif est incorporé à cette étape (thymidine tritiée). (4) Étape de fermeture du brin réparé par ligature de I'ADN nouvellement synthétisé à I'ADN parental. Cette représentation schématique est dérivée des expériences effectuées chez E. coli[19]. On suppose qu'un processus similaire opère dans les cellules de mammifères, bien qu'il n'ait pas été directement démontré.

$\mathrm{m} / \mathrm{s} n^{\circ} 10$ vol. 4, décembre 88 protéines majeures chez $E$. coli et les produits d'une dizaine de gènes différents chez la levure. L'étape limitante de la réparation par excision se situe donc à ce niveau et la réparation de la plupart des cellules mutées dans l'excision-resynthèse sera bloquée à cette étape. Après excision du fragment d'ADN, la discontinuité ainsi formée est comblée par une ADN-polymérase utilisant le brin opposé comme matrice, puis une ADN-ligase permet de lier l'ADN nouvellement synthétisé à l'ADN parental, la molécule ainsi réparée a recouvré son intégrité [8].

En 1968, Cleaver démontra que les fibroblastes isolés de malades XP à forme classique étaient incapables d'effectuer le processus de réparation par excision après irradiation UV [1] Des expériences biochimiques et enzymatiques ont permis de montrer que les cellules XP étaient déficientes dans l'étape de reconnaissance et d'incision du brin lésé. En particulier, l'introduction dans des cellules XP, préalablement irradiées aux UV, d'une enzyme de phage uniquement capable de cliver le brin d'ADN au niveau d'un dimère de pyrimidine permet à ces cellules d'effectuer une réparation d'ADN quasi normale. Ce type d'expérience démontre clairement que l'étape déficiente dans les cellules XP classiques est la première étape du processus de réparation par excision. Ce déficit enzymatique a été retrouvé in vivo sur la peau de malades XP soumise à l'irradiation et dans toutes les cellules isolées de ces malades, y compris les cellules fœetales. Cette caractéristique est utilisée pour la détection anténatale du XP (voir Diagnostic anténatal, p. 616 et figure 7, p. 614).

Différentes méthodes permettent de quantifier l'activité de réparation de l'ADN dans une cellule endommagée: (a) évaluation de la synthèse réparatrice d'ADN correspondant à l'étape de synthèse après excision en présence d'un précurseur radio-actif (thymidine tritiée) soit par mesure directe de la radioactivité incorporée dans l'ADN parental purifié sur gradient de densité, soit par autoradiographie et dénombrement des grains d'argent dans les noyaux des cellules; (b) détermination de la cinétique de perte des lésions sur l'ADN 
en utilisant des sondes spécifiques habituellement immunologiques; (c) mesure de l'incidence des cassures simple brin dans l'ADN cellulaire correspondant à l'efficacité de l'étape d'incision des lésions.

- Groupes de complémentation génétique. La fusion cellulaire entre deux types de fibroblastes provenant d'un malade (A) et d'un malade (B) donne naissance à des cellules hybrides formées des deux noyaux (AB). Si les deux cellules appartiennent au même groupe de complémentation, l'hybride (AB) sera incapable d'assurer la réparation de l'ADN après irradiation UV, habituellement mesurée par autoradiographie. Si les deux cellules appartiennent à deux groupes de complémentation différents, l'hybride (AB) effectuera cette réparation de l'ADN, chaque cellule apportant l'enzyme déficiente dans l'autre (figure 8, p. 615) [9]. Neuf groupes de complémentation génétique (numérotés de $\mathrm{A}$ à I) ont été caractérisés parmi tous les malades XP à forme classique. Chaque groupe de complémentation est totalement ou partiellement déficient dans la réparation par excision (Tableau II) et la sensibilité cellulaire à l'irradiation UV est inversement proportionnelle à l'efficacité de cette réparation (figure 5). Bien que le déficit moléculaire des cellules XP soit encore inconnu, il doit être différent d'un groupe de complémentation à l'autre. Ainsi dans certains groupes, on présume que l'une des enzymes impliquées dans le complexe multienzymatique permettant l'incision de l'ADN lésé est déficiente ou manquante. Une activité résiduelle très faible de réparation de l'ADN résulterait de ce défaut. La complémentation du déficit biochimique de réparation de l'ADN peut aussi être obtenue par micro-injection d'extraits cellulaires de XP (A) dans le noyau d'une cellule XP(B) ou in vitro en mélangeant les deux extraits cellulaires [10]. Cette approche permet d'envisager la purification d'un facteur protéique déficient dans le XP. Actuellement, ni ce facteur protéique ni le gène correspondant n'ont été isolés.

Pour d'autres groupes, le complexe

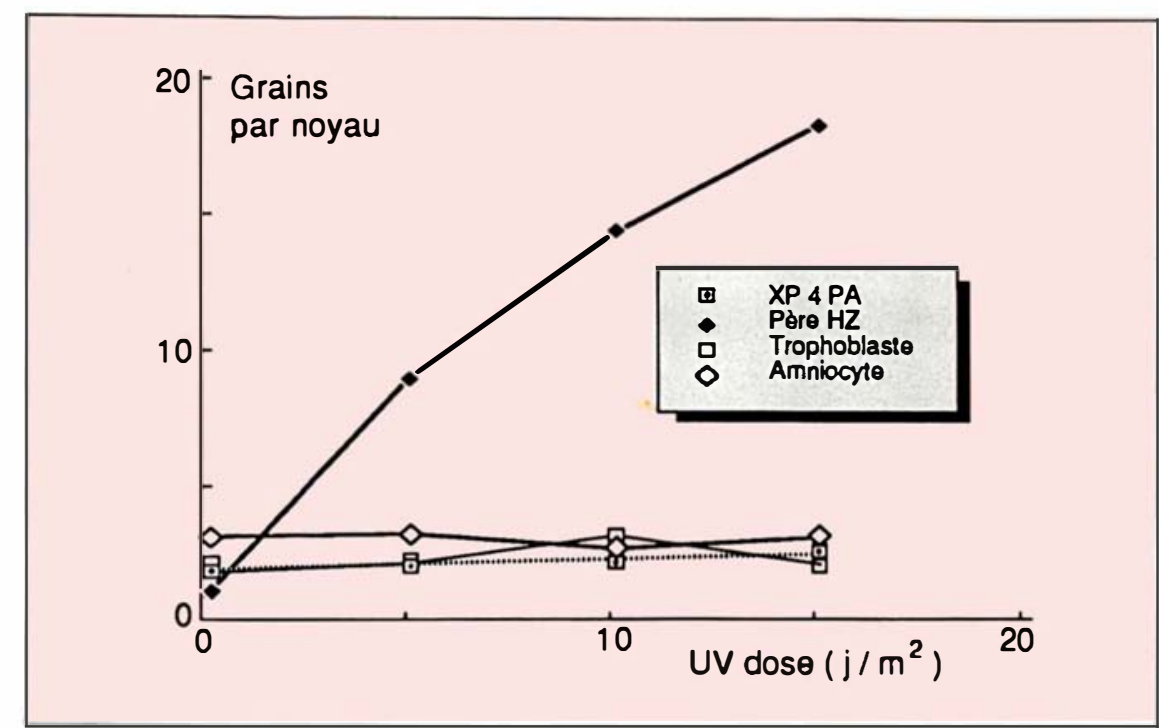

Figure 7. Quantification de la réparation de I'ADN dans une famille à risque en utilisant la technique schématisée dans les figures 6 et 8 . $\checkmark=$ père hétérozygote $(H Z)$ non malade; $\square=$ enfant XP (groupe de complémentation C: XP4PA): biopsie de peau prélevée à quatre ans; $\diamond=$ fœtus $X P$ : prélèvement de liquide amniotique; $\square=$ fœtus XP : prélèvement de trophoblaste.

l'ADN lésé serait présent, mais ne pourrait pas atteindre la lésion, du fait de l'absence d'autres protéines nécessaires à l'accessibilité ou à l'interaction entre enzymes de réparation et ADN. Ainsi un extrait cellulaire isolé de fibroblastes provenant de malades XP appartenant aux groupes A, C ou G, est capable d'éliminer in vitro les dimères de pyrimidine sur de l'ADN nu, mais pas dans la chromatine [11].

En plus du défaut commun aux cellules XP, forme classique, de ne pas réparer l'ADN lésé par les UV ou les agents mimant les UV, chaque groupe de complémentation possède des caractéristiques biochimiques ou enzymatiques particulières (Tableau II). Ainsi le groupe D ne possède qu'une activité partielle en endonucléase capable de réparer les sites abasiques (les AP-endonucléases détectent les sites où il y a eu une perte de bases et clivent le brin d'ADN au niveau de la base perdue. Cette activité représente la première étape de la voie de réparation des sites abasiques). Les protéines qui se lient à l'ADN simple brin ont une activité diminuée dans le groupe $\mathrm{A}$. Les deux malades représentant les groupes $\mathrm{B}$ et $\mathrm{H}$ sont aussi atteints du syndrome de Cockayne (voir plus haut). Les cellules isolées de malade $\mathrm{XP}$ du groupe $\mathrm{C}$ possèdent une activité normale de réparation de l'ADN sur la partie du génome cellulaire liée à la matrice nucléaire, partie probablement en réplication ou en transcription intense. Le reste de l'ADN génomique n'étant pas réparé du tout [4]. Cette particularité du groupe $\mathrm{C}$ est intéressante, car elle rappelle le mode de réparation préférentielle trouvé dans les cellules de rongeurs, où les gènes en cours de transcription et qui apparaissent nécessaires à la vie cellulaire sont réparés plus rapidement et plus efficacement que le reste de l'ADN cellulaire [12].

- XP variant. Environ $20 \%$ des malades XP possèdent des cellules légèrement plus sensibles aux UV 
que les cellules normales et une activité de réparation par excision-resynthèse tout à fait normale. Cette forme dite variante est cliniquement comparable à la forme classique, mais les signes cliniques et en particulier les tumeurs cutanées malignes apparaissent beaucoup plus tardivement, entre 15 et 40 ans.
Le défaut biochimique responsable du XP variant se situe au niveau dela tolérance des lésions au moment de la réplication de l'ADN. La réplication est en général bloquée de façon transitoire par les lésions induites par les UV et l'ADN nouvellement synthétisé possède une faible masse moléculaire juste après l'irradiation

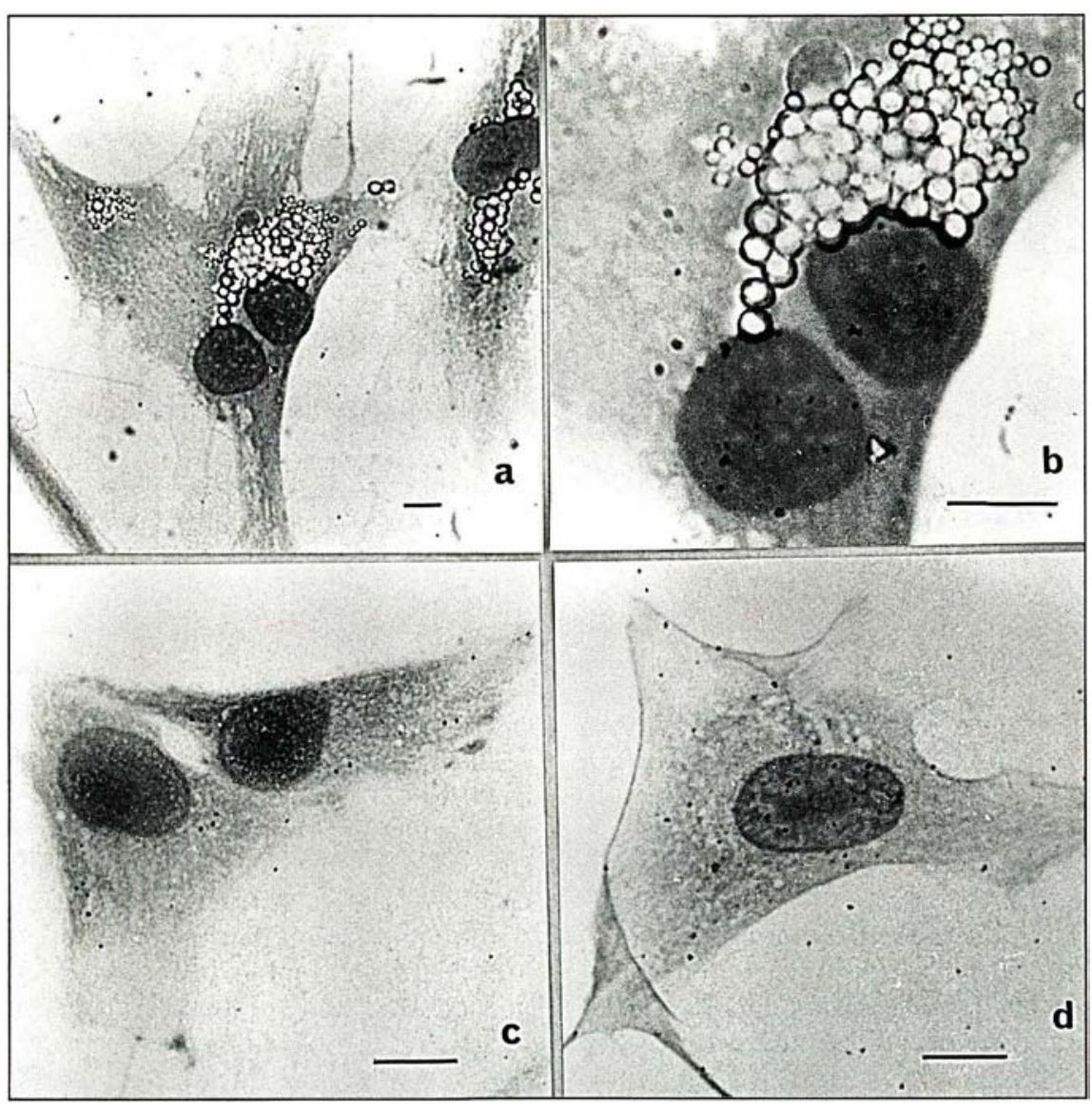

Figure 8. a et b. Autoradiographie d'un hybride cellulaire entre des cellules XP groupe $A$ et XP groupe $C$ montrant la réparation de I'ADN irradié $\left(20 \mathrm{~J} / \mathrm{m}^{2}\right.$, UV $254 \mathrm{~nm}$ ) et en présence de thymidine tritiée (a $\times 600 ; b$ x 1900). Les cellules XPA contiennent dans leur cytoplasme des billes de latex de 1,5 $\mu \mathrm{m}$ de diamètre et les cellules XPC des billes de $3 \mu \mathrm{m}$ de diamètre Les hybrides (AC) formés sous l'action du polyéthylène glycol contiennent les deux types de billes dans leur cytoplasme. La présence de grains d'argent dans le noyau des cellules indique une activité de réparation de l'ADN, schématisée dans l'étape de resynthèse de la figure 6 . $\boldsymbol{c}$ et $\boldsymbol{d}$. Autoradiographie de cellules fotales irradiées aux UV in vitro dont la réparation est quantifiée par la présence de grains d'argent correspondant à l'incorporation de thymidine tritiée (cellules trophoblastiques cultivées à partir de biopsies de trophoblaste prélevées à la $8^{e}$ semaine de grossesse). $c=$ cellules de fœtus XP incapable de réparer I'ADN (x 1500); $d=$ cellules de fœtus témoin capable de réparer I'ADN (x 1500); les barres horizontales représentent $10 \mu \mathrm{m}$.
UV. Dans une cellule normale, l'étape dite de réparation post-réplicative restitue la masse moléculaire normale de l'ADN dans les quelques heures qui suivent l'irradiation. Ce processus est déficient dans les cellules de XP variant et la taille de l'ADN reste faible pendant une plus longue durée [13]. Cette étape est spécifiquement inhibée par la caféine dans les cellules XP variants. Bien que le mécanisme moléculaire de cette étape soit encore inconnu, on imagine qu'elle pourrait être due à des enzymes permettant de tolérer la présence de lésions. Ces enzymes pourraient être inductibles dans les cellules normales et être inactives dans les XP variants.

- Mutagenèse. Les cellules en culture provenant de malades XP classiques ou variants expriment une fréquence de mutation beaucoup plus grande que les cellules normales après irradiation ultraviolette ou traitement par des cancérogènes chimiques. Les anomalies pigmentaires observées in vivo sur la peau exposée ont été interprétées comme étant dues à la prolifération d'un clone cellulaire muté. Ce taux élevé de mutagenèse détecté in vitro sur plusieurs locus et la fréquence particulièrement importante de cancers cutanés détectés in vivo dans les zones exposées au soleil sont probablement les meilleurs arguments pour étayer la théorie selon laquelle la mutation somatique est à l'origine du cancer. En effet, le XP est le meilleur exemple chez l'homme d'une corrélation entre la présence de lésions non réparées sur la molécule d'ADN, le taux élevé de mutations induites et la fréquence énorme de tumeurs précoces. Cette hypothèse a été récemment vérifiée par l'isolement d'oncogènes cellulaires activés par une mutation ponctuelle dans des tumeurs cutanées fraîchement isolées de malades $\mathrm{XP}[14,15]$.

- Activité catalase. Les catalases sont des enzymes capables de dismuter l'eau oxygénée en eau et oxygène. L'eau oxygénée est produite soit au cours du métabolisme cellulaire normal, soit lors de la transformation des radicaux oxygène libres formés sous l'action des rayonnements ionisants. Ces radicaux libres ainsi que 


\begin{tabular}{|c|c|c|c|c|}
\hline \multicolumn{5}{|c|}{$\begin{array}{l}\text { Tableau II } \\
\text { INIQUES ET BIOCHIMIQUES DES DIFFÉRENTS GROUPES } \\
\text { MENTATION DU XERODERMA PIGMENTOSUM(1) }\end{array}$} \\
\hline $\begin{array}{l}\text { Groupe de } \\
\text { complémentation }\end{array}$ & $\begin{array}{l}\text { Fréquence } \\
\text { relative (2) } \\
\text { (\%) }\end{array}$ & $\begin{array}{l}\text { Efficacité de } \\
\text { réparation par } \\
\text { excision après UV } \\
\text { mesurée par } \\
\text { autoradiographie } \\
\text { (\% par rapport } \\
\text { aux témoins) }\end{array}$ & $\begin{array}{l}\text { Anomalies } \\
\text { neurologiques }\end{array}$ & Spécificité biochimique \\
\hline A & 29 & $\leqslant 5$ & $+/-$ & $\begin{array}{l}\text { Activité faible en protéine se liant à } \\
\text { I'ADN simple brin }\end{array}$ \\
\hline B & 0,5 & $3-7$ & + & Associé à un syndrome de Cockayne \\
\hline C & 27 & $10-25$ & $+1-$ & $\begin{array}{l}\text { Réparation préfentielle de I'ADN lié } \\
\text { à la matrice nucléaire }\end{array}$ \\
\hline D & 15 & $25-50$ & + & $\begin{array}{l}\text { Activité partielle en Ap-endo- } \\
\text { nucléase } \text { (4) }^{-1}\end{array}$ \\
\hline$E$ & 1 & $40-60$ & - & - \\
\hline$F$ & 2 & $10-20$ & - & - \\
\hline G & 1 & 5 & + & - \\
\hline $\mathrm{H}$ & 0,5 & 30 & + & Associé à un syndrome de Cockayne \\
\hline 1 & 0,5 & $15-25$ & $?$ & - \\
\hline Variant & 24 & 100 & - & $\begin{array}{l}\text { Anomalie au niveau de la réparation } \\
\text { post-réplicative } \\
\text { Apparition tardive des symptômes }\end{array}$ \\
\hline
\end{tabular}

(1) Reproduit d'après [4 et 6]. (2) D'après une étude portant sur 188 malades. (3) $+/-$ signifie présence d'anomalies dans une fraction des malades. (4) Les Ap-endonucléases sont des enzymes qui détectent les sites abasiques où il y a eu une perte de base et clivent le brin d'ADN au niveau de la base perdue. Cette activité représente la première étape de la voie de réparation des sites abasiques.

l'eau oxygénée sont des substances très toxiques pour la cellule qui induisent en particulier des lésions et des cassures simple brin sur l'ADN. Les cellules de XP, quel que soit le type génétique étudié, sont fortement déficientes en activité catalase et donc dans leur capacité d'éliminer l'eau oxygénée[16]. L'impossibilité de cette détoxication pourrait expliquer les anomalies neurologiques progressives de certains malades XP, désordres qui seraient alors dus à l'accumulation de substances toxiques d'origine cellulaire dans les cellules nerveuses. L'accumulation de lésions ainsi induites pourrait aussi être à l'origine des tumeurs cérébrales observées dans le XP.

\section{Diagnostic anténatal}

Le XP étant une maladie transmise de façon autosomique récessive, il

naître un enfant atteint à chaque nouvelle grossesse, dans une famille à risque. Dans ces conditions, il est concevable de pratiquer un diagnostic prénatal pour chaque nouvelle naissance.

Des cellules fœtales sont cultivées in vitro à partir de biopsies de trophoblastes (prélevées dans la $10^{e}$ et la $11^{e}$ semaine) ou de liquide amniotique prélevées vers la $16^{\mathrm{e}}$ semaine d'aménorrhée). Après différentes doses d'irradiation UV, les cellules sont incubées en présence de thymidine tritiée et la réparation par excision-resynthèse est quantifiée en comptant le nombre de grains d'argent par noyau après autoradiographie (figures 7 et 8). La réussite du diagnostic prénatal repose sur la possibilité de pouvoir différencier de façon significative la réparation par excision entre les parents hétérozygotes et le cas index. Ceci implique d'avoir comparé, avant le test, les efficacités de répara- tion de l'ADN des cellules provenant de biopsies de peau des membres de la famille. Ce test ne permet de détecter que les enfants XP à forme classique. La mesure de la réparation des cellules fœtales permet alors facilement de les classer comme identiques aux cellules parentales ou identiques aux cellules de l'enfant XP. Pour un fœtus XP donné, nous avons trouvé des efficacités de réparation similaires entre les cellules d'origine villositaire et amniotique (figure 7). Le seul artefact possible que nous ayons rencontré porte sur des cellules contaminées par des mycoplasmes, probablement au moment du prélèvement. Dans ces conditions, il existe un bruit de fond considérable de grains d'argent dans le cytoplasme cellulaire dû au métabolisme spécifique des mycoplasmes qui masque le taux réel de réparation cellulaire intranucléaire. L'interprétation des résultats du test est alors délicate. 
En ce qui concerne la forme XP variante, le test prénatal a déjà été proposé [17], mais par l'utilisation une technique biochimique différente. En effet, après irradiation ultraviolette des cellules fœtales et incorporation de thymidine tritiée afin de marquer l'ADN nouvellement synthétisé, la taille de celui-ci est alors examinée en fonction du temps sur gradient de saccharose. Des cellules normales seront capables de produire de l'ADN de haut poids moléculaire après UV, plus rapidement que des cellules de XP variant. La comparaison de cette cinétique entre cellules fœtales et cellules parentales permet donc de déterminer si le fœtus appartient au groupe $\mathrm{XP}$ variant.

La gravité extrême du XP et l'absence totale de traitement efficace plaident en faveur de l'utilisation systématique du diagnostic anténatal. Son développement actuel par biopsie de trophoblaste, prélevée précocement au cours de la grossesse, permet de proposer un avortement thérapeutique dans des conditions acceptables. Le clonage et la caractérisation des gènes responsables du $\mathrm{XP}$ sont en cours [18]. Il est évident que la caractérisation moléculaire de ces gènes pourra faciliter et rendre plus rapide le diagnostic prénatal et pourrait éventuellement permettre d'envisager une thérapie génique

\section{Remerciements}

Nous tenons à remercier A. Benoît et A. Margot pour leur excellente aide technique. Nous remercions également le Dr. K.H. Kraemer pour nous avoir procuré les données statistiques d'origine américaine. Ce travail été soutenu par l'ARC, la LFNCC, le GEFLUC et la CEE (contrat Bio-163 F).

\section{TIRÉS A PART}

\section{A. Sarasin.}

$\mathrm{m} / \mathrm{s} n^{\circ} 10 \mathrm{vol}$. 4, décembre 88

\section{Summary}

Xeroderma pigmentosum is a rare disease transmitted with an autosomal recessive inheritance. Patients exhibit extreme sunsensitivity, leading to epitheliomas in exposed skin. The median age for the appearance of skin cancers among Xeroderma pigmentosum patients is 8 years, more than 50 years earlier than for the general population. Xeroderma pigmentosum cells are deficient in DNA repair either in the excisionresynthesis pathway (classical form) or in the post-replicative pathway (variant form). The genetic complexity in this disease is attested by the existence of ten complementation groups showing some specific defects. This paper reviews the clinical, biochemical and genetical characteristics of Xeroderma pigmentosum. This disease is a model system to understand the initiation and the progression of skin tumors in relation with the presence of unrepaired DNA damages. This is the first human disease for which it has been established that DNA lesions on the cellular genome are directly responsible for the induction of cancers. We report in this paper our results concerning the prenatal diagnosis of Xeroderma pigmentosum in families at risk. Quantification of UV-induced DNA repair is carried out on cultured cells isolated from amniotic fluids or chorionic biopsies. Comparison of the efficiency of DNA repair between fœtal cells and both the heterozygous parental cells, normal for repair, and the affected sibling cells, deficient for repair, tells us the medical state of the fotus.

FLASH. Des cellules appartenant au groupe de complémentation de Xeroderma pigmentosum sont déficients en une protéine nucléaire qui reconnaît normalement I'ADN lésé. Source: Science, 28 octobre 1988, p. 564. 